\title{
BMJ Open Protocol for a nationwide prospective, observational cohort study of foreign- body airway obstruction in Japan: the MOCHI registry
}

\author{
Tatsuya Norii (D) , ${ }^{1,2}$ Yutaka Igarashi, ${ }^{3}$ Kim Sung-Ho, ${ }^{4}$ Shimpei Nagata, ${ }^{5}$ \\ Takashi Tagami, ${ }^{6}$ Yudai Yoshino, ${ }^{7}$ Takuro Hamaguchi, ${ }^{6}$ Riko Maejima, ${ }^{8}$ \\ Shunichiro Nakao, ${ }^{2}$ Danielle Albright, ${ }^{1}$ Shoji Yokobori, ${ }^{3}$ Hiroyuki Yokota, ${ }^{3}$ \\ Takeshi Shimazu, ${ }^{2}$ Cameron Crandall ${ }^{1}$
}

To cite: Norii T, Igarashi Y, Sung-Ho K, et al. Protocol for a nationwide prospective, observational cohort study of foreign-body airway obstruction in Japan: the $\mathrm{MOCH}$ registry. BMJ Open 2020;10:e039689. doi:10.1136/ bmjopen-2020-039689

- Prepublication history for this paper is available online. To view these files, please visit the journal online (http://dx.doi. org/10.1136/bmjopen-2020039689).

Received 22 April 2020 Revised 09 June 2020 Accepted 10 June 2020

Check for updates

(c) Author(s) (or their employer(s)) 2020. Re-use permitted under CC BY-NC. No commercial re-use. See rights and permissions. Published by BMJ.

For numbered affiliations see end of article.

Correspondence to

Dr Tatsuya Norii;

tanorii@salud.unm.edu

\section{ABSTRACT}

Introduction Foreign body airway obstruction (FBAO) is a major public health issue worldwide. In 2017, there were more than 5000 fatal choking cases in the USA alone, and it was the fourth leading cause of preventable injuryrelated death in the home and community. In Japan, FBAO is the leading cause of accidental death and with almost 9000 fatalities annually. However, research on FBAO is limited, particularly on the impact of a foreign body (FB) removal manoeuvres by bystanders. The primary objective of this study is to determine the impact of bystander FB removal manoeuvres on 1 month neurological outcome. Our secondary objectives include (1) evaluating the efficacy of a variety of FB removal manoeuvres; (2) identifying risk factors for unsuccessful removal and (3) evaluating the impact of time intervals from incidents of FBAO to FB removal on neurological outcome.

Methods and analysis We will conduct a nationwide multi-centre prospective cohort study of patients with FBAO who present to approximately 100 emergency departments in both urban and rural areas in Japan. Research personnel at each participating site will collect variables including patient demographics, type of FB and prehospital variables, such as bystander FB removal manoeuvres, medical interventions by prehospital personnel, advanced airway management and diagnostic findings. Our primary outcome is 1 month favourable neurological outcome defined as cerebral performance category 1 or 2 . Our secondary outcomes include success of FB removal manoeuvres and complications from the manoeuvres. We hypothesise that bystander FB removal manoeuvres improve patient survival with a favourable neurological outcome.

Ethics and dissemination This study received research ethics approval from Nippon Medical School Hospital (B-2019-019). Research ethics approval will be obtained from all participating sites before entering patients into the registry. The study was registered at the University Hospital Medical Information Network (UMIN) Clinical Trials Registry. Trial registration number UMIN 000039907.

\section{INTRODUCTION}

Contrary to the belief that foreign-body airway obstruction (FBAO) is a 'rare' cause

\section{Strengths and limitations of this study}

- Our study will be the first large-scale prospective study of patients with foreign-body airway obstruction (FBAO).

- This study will evaluate the impact of bystander foreign body (FB) removal manoeuvres on 1 month favourable neurological and include enough cases to adjust for prespecified confounders, such as patients' age, witness of the event and type of FBAO.

- The study will also provide data on the effectiveness of $\mathrm{FB}$ manoeuvres in the real world.

- Although this study will include multiple emergency departments throughout Japan, the results of the study might not be applicable to other countries where the population and healthcare system are different.

of cardiac arrest, FBAO is a major public health issue worldwide. In 2017, there were more than 5000 fatal cases due to choking in the USA alone, and it was the fourth leading cause of preventable injury-related death in the home and community. ${ }^{1}$ Similar to the USA, death or morbidity from FBAO has received increasing attention in many developed countries in Europe and Asia. ${ }^{2-5}$ In Japan where life expectancy is the longest in the world, FBAO has been the leading cause of accidental death since 2006, with almost 9000 fatal cases reported every year in a country with 126 million habitants. ${ }^{6}$

Since elderly people are at high risk of FBAO, fatality due to FBAO will most likely continue to increase in many countries. Despite the need for further studies on FBAO, research on FBAO is still limited. No major changes on FBAO management have been made for nearly 15 years in the resuscitation guidelines from the American Heart 
Association and the International Liaison Committee on Resuscitation.

In contrast to the well-studied impact of bystander cardiopulmonary resuscitation (CPR) on survival and neurological outcomes in patients with out-of-hospital cardiac arrest, ${ }^{78}$ little is known about the effectiveness of bystander attempts of airway foreign body (FB) removal manoeuvres, such as a back blow, the abdominal thrust (ie, Heimlich manoeuvre) and how these attempts affect outcomes in patients with FBAO. Additionally, these manoeuvres occasionally cause a major injury, and the incidence of complications has not been well studied. ${ }^{9}$ Thus, a large-scale registry of FBAO that includes timing and type of FB removal attempts, their successes and complications is warranted.

\section{OBJECTIVES}

The primary objective of this study is to determine the impact of bystander FB removal manoeuvres on 1 month favourable neurological outcome defined as Cerebral Performance Category (CPC) 1 (good recovery) or 2 (moderate disability) among patients with FBAO ${ }^{10}$ Our secondary objectives include $(1$,$) evaluating the efficacy$ of a variety of FB removal manoeuvres; (2,) identifying risk factors for unsuccessful removal and (3,) evaluating the impact of time intervals from incidents of FBAO to FB removal on favourable neurological outcome.

\section{METHODS AND ANALYSIS}

\section{Study design}

The MOCHI (uulti-center Observational CHoking Investigation) registry is a prospective, nationwide, observational study that consists of all patients who presented to emergency departments (EDs) with FBAO in Japan. We define FBAO as life-threatening mechanical airway obstruction caused by FB in the airway. We will include all (adult and paediatric) patients who present to the ED with FBAO from April 2020 to March 2023. We will exclude patients who present with the aspiration of sputum or gastric contents, choking with gas, drowning, unwitnessed cardiac arrest, loss of consciousness before FBAO, strangulation or a neck tumour that caused suffocation. Research ethics approval will be obtained from all participating sites before entering patients into the registry. The requirement of written informed consent was waived.

\section{Study setting}

In Japan, emergency medical dispatchers typically instruct bystanders to perform basic FB removal procedures via phone. ${ }^{11}$ There is some variation among emergency medical system (EMS) protocols on how to instruct FB removal procedures to a layperson. However, dispatchers typically instruct callers to attempt a back blow and the abdominal thrust manoeuvre, or combinations of these manoeuvres. In the field, protocols recommend EMS personnel encourage a patient to cough, perform a back blow and the abdominal thrust manoeuvre. When a patient becomes unresponsive or suffers from cardiac arrest, EMS personnel perform chest compressions and attempt to remove the FB. Advanced airway management, including supraglottic airway device placement or endotracheal intubation, is performed by specially trained EMS personnel. Depending on the severity of incidents and availability, doctor-staffed ambulances are also dispatched and emergency physicians provide immediate procedures for critically ill patients at the scene. Because of the very strict criteria for discontinuation of resuscitation efforts in the field, it is extremely rare for EMS to not transport patients to the ED.

\section{Data collection and quality control}

Research personnel at each participating site will collect variables including patient demographics, type of $\mathrm{FB}$, prehospital variables such as FB removal manoeuvres performed by bystanders, other medical interventions by prehospital personnel such as advanced airway management, and in-hospital diagnostic findings and treatments including core body temperature management and extracorporeal membrane oxygenation. We will also record timing of key events, such as the onset of FBAO, attempts of rescue procedures, EMS arrival on the scene, advanced airway management, resolution of obstruction and the onset of cardiac arrest (if any). In regards to FB removal manoeuvres, a patient's body position (eg, a prone or head-down position) will also be recorded since several studies have shown the effectiveness of certain positions. $^{12} 13$

The registry will also record the type of airway FBs and their locations. Most of the previous large-scale studies on FBAO retrospectively analysed cardiac arrest registries, which often lack key variables for FBAO, such as bystanders' efforts to remove FB, type of FB and locations of FBs. For this reason, there has previously been no formal classification system available for FBAO. Due to the heterogeneity of FBAO victims and absence of classifications of FBAO, we developed a new classification system, called MOCHI classification in 2018 and published the proposal in 2019. ${ }^{14}$

Figure 1 summarises the MOCHI classification system. In the classification system, FBAO cases are classified into three types based on anatomical and physiological characteristics: type 1, upper airway obstruction by a large FB that cannot pass through the vocal cord or a large amount of residue that completely obstructs the airway; type 2 , trachea or bilateral main bronchus obstruction by an FB or a large amount of residue and type 3 , unilateral bronchus or distal bronchus obstruction by an FB, residue or liquid. ${ }^{14}$ This classification system allows researchers to report FBAO cases more consistently, and the system has already been cited by an article on FBAO.$^{15}$ Variables for FB characteristics would be helpful to examine whether certain characteristics are associated with unsuccessful FB removal or not. For example, some food materials, such 


\begin{tabular}{|c|c|c|c|c|}
\hline Type & \multicolumn{2}{|c|}{ Anatomy } & Physiology & Definition \\
\hline 1 & & $\begin{array}{l}\text { Oral cavity, } \\
\text { pharynx, or } \\
\text { larynx } \\
\text { (blue) }\end{array}$ & Possible & $\begin{array}{l}\text { Upper airway obstruction by a large foreign body that } \\
\text { cannot pass through the vocal cord or a large amount of } \\
\text { residue that completely obstructs the airway. }\end{array}$ \\
\hline 2 & & $\begin{array}{l}\text { Trachea } \\
\text { above } \\
\text { carina } \\
\text { (light blue) }\end{array}$ & obstruction & $\begin{array}{l}\text { Trachea and/or bilateral main bronchus obstruction by a } \\
\text { foreign body or a large amount of residue. }\end{array}$ \\
\hline 3 & & $\begin{array}{l}\text { Bronchi, } \\
\text { bronchioles, } \\
\text { and lungs } \\
\text { (white) }\end{array}$ & $\begin{array}{l}\text { Partial } \\
\text { obstruction }\end{array}$ & $\begin{array}{l}\text { Unilateral bronchus and/or distal bronchus obstruction } \\
\text { by a foreign body, residue, or liquid. }\end{array}$ \\
\hline
\end{tabular}

Figure 1 'MOCHI' classification of the location of foreign bodies in airway obstruction. MOCHI, Multi-center Observational CHoking Investigation.

as a sticky rice cake or others might be associated with better or worse outcomes. ${ }^{16}$ For this reason, type of FB, including the type of food material, will be recorded. In order to assist classification consistency and accuracy, we developed a MOCHI classification algorithm (figure 2) and provided bronchoscopic and CT images that demonstrated classic examples (figures 3-6).

We are also interested in the incidence and severity of complications from rescue procedures for FBAO. Although rare, multiple case reports have described severe and sometimes fatal injuries that were caused by the Heimlich manoeuvre. ${ }^{17-19}$ van der Ham et a ${ }^{19}$ published a report describing a list of cases who had severe complications from abdominal thrust manoeuvre. The list included several cases who died due to the complications (eg, peritonitis after gastric perforation). However, due to the lack

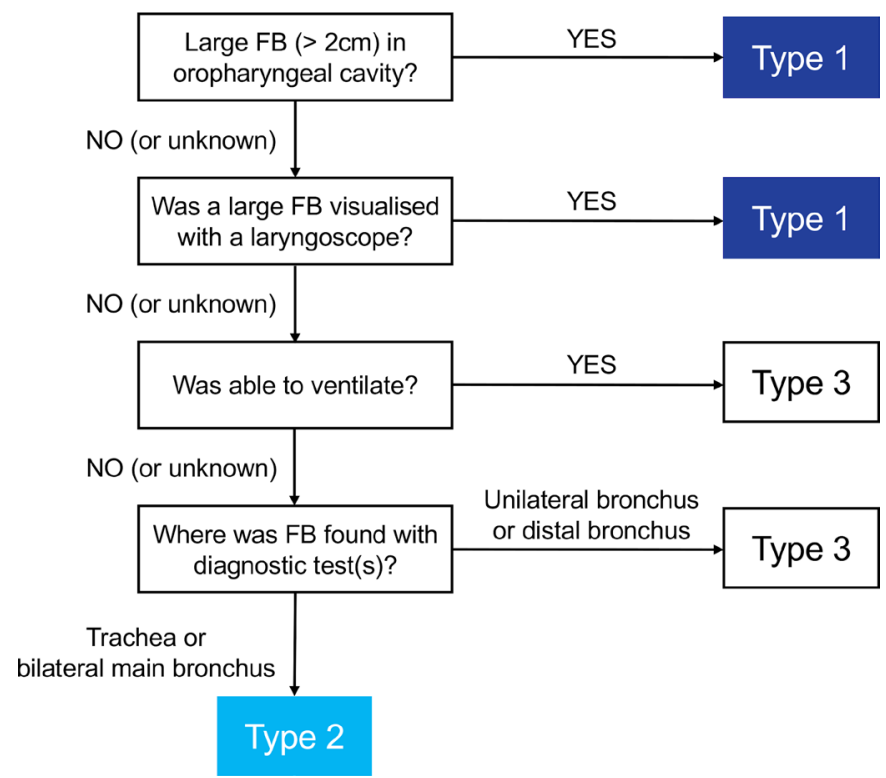

Figure $2 \mathrm{MOCH}$ algorithm. FB, foreign body; $\mathrm{MOCHI}$, Multi-center Observational CHoking Investigation. of large-scale studies for FBAO, complication rates from rescue manoeuvres are still largely unknown. To begin to address this gap, our registry includes potential complications that are found with diagnostic tests, such as CT scan, postmortem imaging study and autopsy.

Our primary outcome is 1 month favourable neurological outcome defined as CPC 1 (good recovery) or 2 (moderate disability). ${ }^{10}$ Our secondary outcomes include the success of FB removal manoeuvres and complications from the manoeuvres. We hypothesise that bystander FB removal manoeuvres improve patient survival with a favourable neurological outcome.

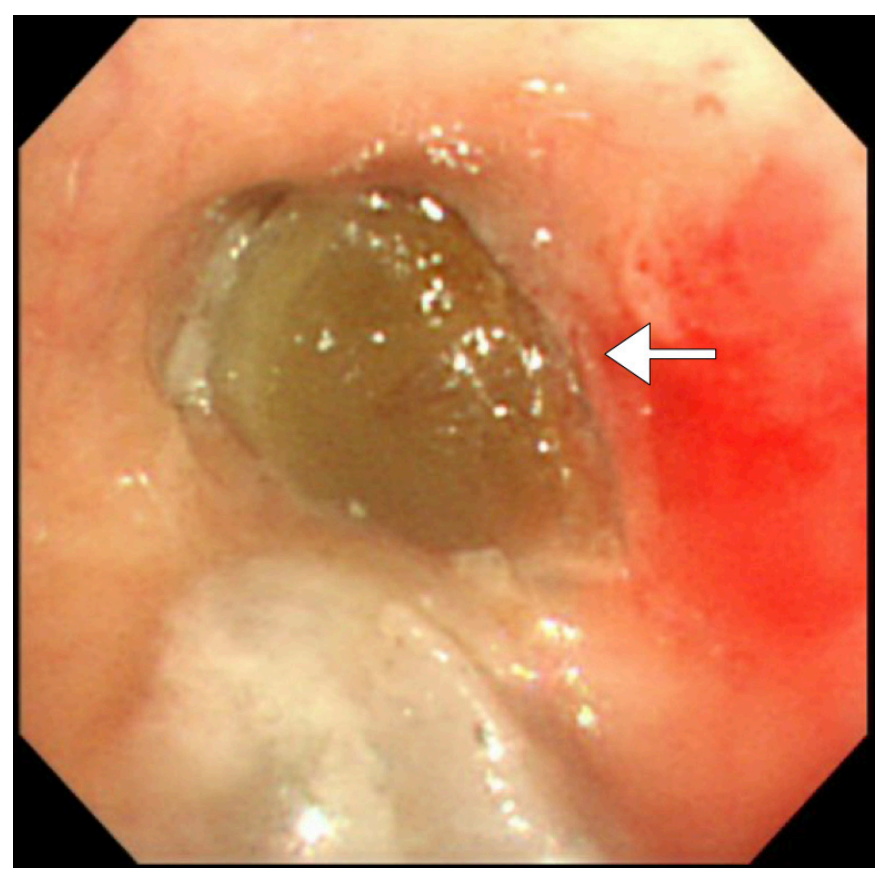

Figure 3 An example of type $3 \mathrm{MOCH}$ airway foreign body. A bronchoscopic image demonstrates that a food material is completely obstructing left main bronchus (arrow). $\mathrm{MOCHI}$, Multi-center Observational CHoking Investigation. 


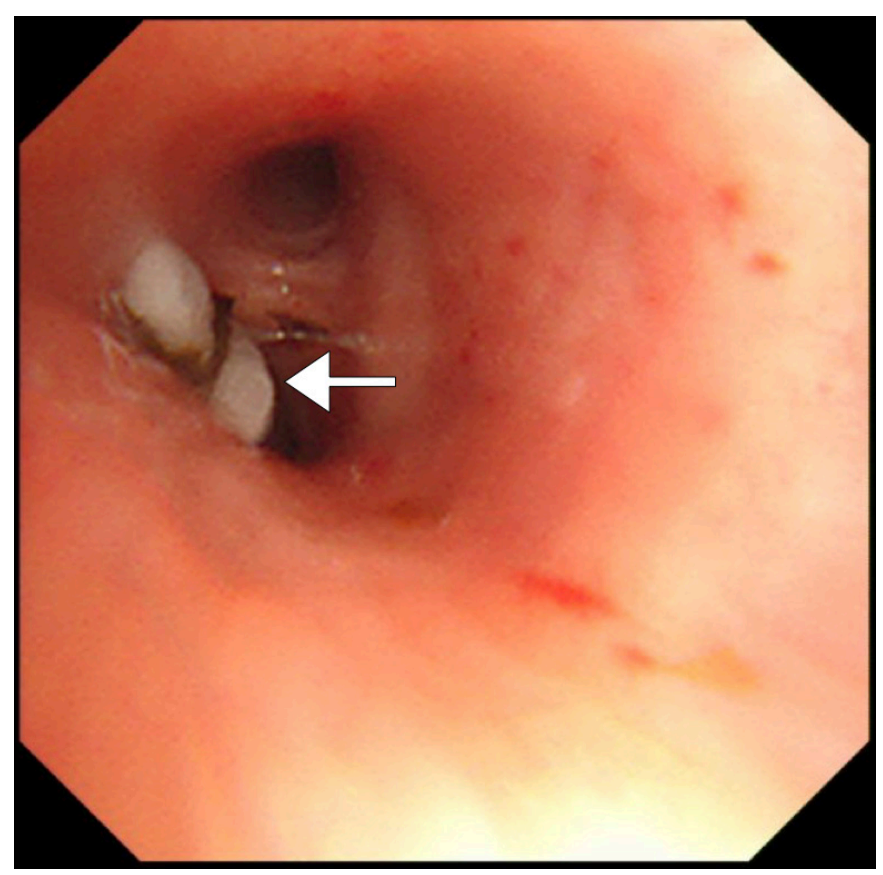

Figure 4 An example of type $3 \mathrm{MOCHI}$ airway foreign body. A bronchoscopic image demonstrates pieces of food material (arrow) in one of the peripheral bronchioles. MOCHI, Multicenter Observational CHoking Investigation.

\section{Statistical analysis}

Sample size estimation

Based on our previous retrospective study of eight EDs that included 385 cases over 5 years $(9.6$ cases/site/year as average), we plan to recruit a minimum of 30 EDs to collect data on approximately 10 cases per site per year for a total of at least 1000 cases across all sites in 3 years.

In order to compare patients who receive bystander FB removal manoeuvres to those who do not, we will use multiple logistic regression analyses for our primary statistical analysis, adjusting for the following seven prespecified covariates, including age (years), sex (male/female), activity of daily life (independent/ dependent), incident in a public location (public/ private residence), witness (yes/no), MOCHI classification (type 1/2/3) and EMS response time (time from call to dispatch centre to EMS arrival on the scene in minutes). Age and EMS response time will be treated as continuous variables, and the rest of the variables will be treated as dichotomous.

Sample size was calculated based on a similar previous study $^{20}$ and the general rule that a model must fit no more than $\mathrm{m} / 15$ parameters to allow for proper multivariable analysis and to be generalisable to future patients, where $\mathrm{m}$ is the effective sample size. Therefore, the minimum number of cases required for the model will be 135 with $9 \mathrm{df}$ ( $2 \mathrm{df}$ for continuous variables, $1 \mathrm{df}$ for dichotomous). A multivariable model with a complexity of $9 \mathrm{df}$ can be reliably fitted when the effective sample size is at least $9 \times 15=135$ subjects.

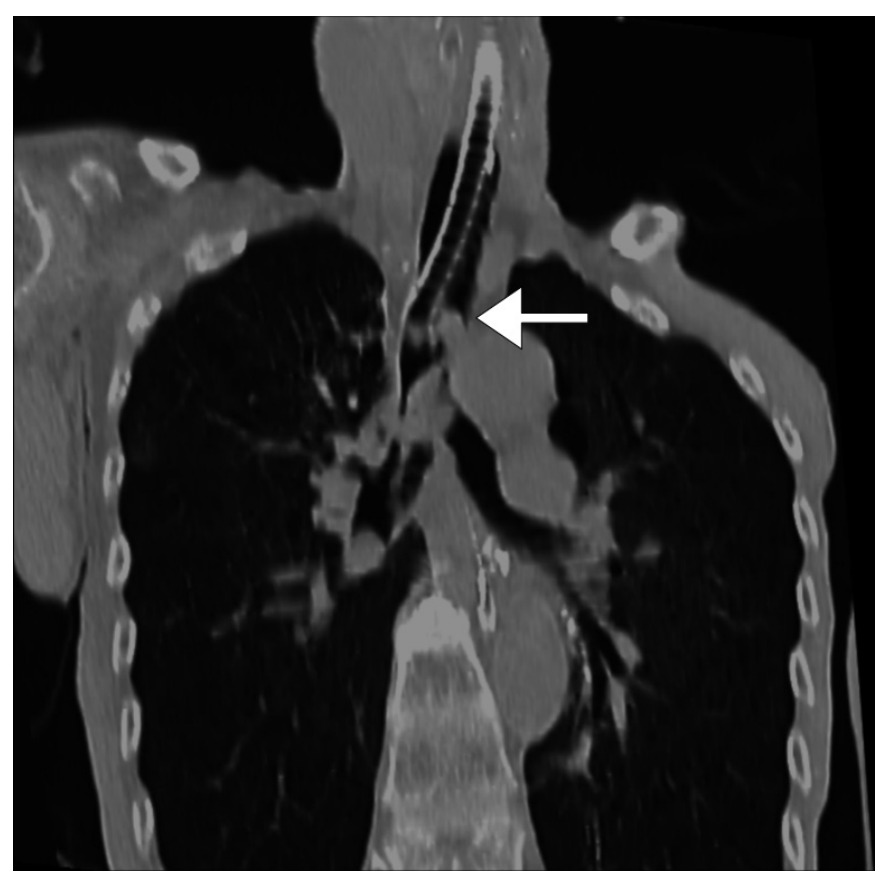

Figure 5 An example of type $2 \mathrm{MOCH}$ airway foreign body. A coronal CT image demonstrates a large airway foreign body in trachea (arrow). Note that the foreign body is pushing an endotracheal tube to side. $\mathrm{MOCHI}$, Multi-center Observational CHoking Investigation.

\section{Strengths and limitations of the study design}

Our study will be the first large-scale prospective study of patients with FBAO. This study will characterise patients with FBAO. We aim to evaluate the impact of bystander FB removal manoeuvres on survival with a favourable neurological outcome among patients who suffer from FBAO. Since this study is an observational study, we can examine the correlation but not causality. However, our

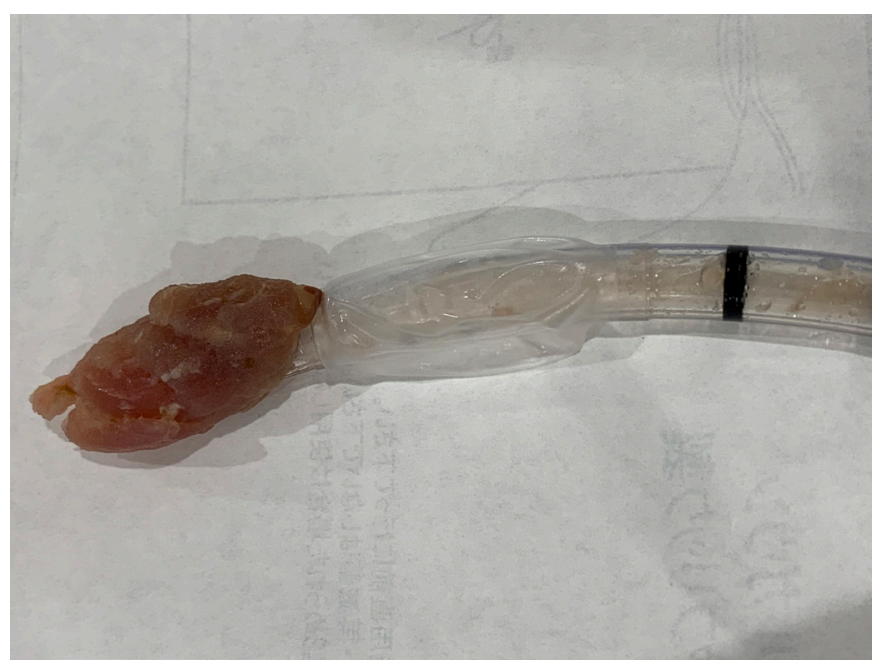

Figure 6 An example of type $2 \mathrm{MOCH}$ airway foreign body. A large foreign body (a piece of tuna fish) was found in the trachea and removed together with the endotracheal tube due to the size of the piece that was larger than the diameter of intubation tube $(7.5 \mathrm{~mm})$. MOCHI, Multi-center Observational CHoking Investigation. 
study will include enough cases to adjust for pre-specified confounders, such as patients' age, the witness of the event and type of FBAO. Although this study will include multiple EDs throughout Japan, the results of the study might not be applicable to other countries where the population and healthcare systems are different.

\section{Patient and public involvement}

No patient or public involvement.

\section{DISCUSSION}

Bystander CPR and defibrillation using an automated external defibrillator (AED) play a significant role, if not the most important, in out-of-hospital cardiac arrest. The recent study conducted by Riva $e t$ al in Sweden revealed that there has been a significant increase of bystander CPR since 2010 and the chance of survival was almost doubled with bystander CPR compared with no CPR before EMS arrival. ${ }^{21}$ In contrast, the association between bystander attempts at FB removal and FBAO victim survival and neurological outcomes has not been well studied. In the MOCHI study, we seek to determine the impact of bystander's FB removal manoeuvres on survival and favourable neurological outcomes. We hypothesise that a bystander FB manoeuvre is associated with survival and a favourable neurological outcome compared with no FB removal attempt before EMS arrival.

FBAO and drowning share similar pathophysiology. In both conditions, the central problem is asphyxia (ie, sudden loss of oxygen supply that is followed by hypoxia in major organs, including the brain). Unless the condition is treated immediately and adequately, brain damage from prolonged hypoxemia causes severe neurological sequelae. The chance of favourable neurological outcome (defined as CPC of 1 or 2) is less than $3 \%$ for patients who suffer from out-of-hospital cardiac arrest due to FBAO.$^{22}{ }^{23}$ These patients tend to have a lower chance of favourable neurological outcome compared with cardiac arrest patients from other causes.

The priorities of resuscitation efforts are also similar in both conditions. Removal of mechanical obstructions that prohibit oxygen from flowing to lungs (ie, water in submersion, FB in FBAO) is the most important step for both conditions. In drowning victims, multiple studies have shown the impact of duration of submersion and water temperature on outcomes. ${ }^{24-26}$ Risk of death or severe neurological sequelae is only $10 \%$ in drowning victims if the duration of submersion is less than $5 \mathrm{~min}$. The risk of poor outcome increases to $56 \%, 88 \%$ and nearly $100 \%$ if the duration is $5-10 \mathrm{~min}, 11-25 \mathrm{~min}$ and $>25$ min, respectively. ${ }^{25}$ However, little is known about the association between duration of FBAO (ie, the time interval between the onset of choking and successful removal of $\mathrm{FB}$ ) and survival and neurological outcomes.

We investigated the association between the duration of FBAO and outcomes in two previous retrospective cohort studies. The first study was a single-centre retrospective cohort study that included 137 patients over a period of 7 years. This study showed a significant relationship between FB removal by a bystander and favourable neurological outcome. ${ }^{27}$ Our more recent study included 386 patients in eight EDs showed that almost half of the patients had favourable neurological outcome (CPC 1 or 2) if FBAO was resolved less than $5 \mathrm{~min}$, but the rate dropped to $28 \%$ if FBAO was not successfully treated until 6-10 min. However, the studies included a relatively small number of patients, and there was also a large number of patients with missing time data due to the retrospective design. Therefore, the association between duration of FBAO and survival and neurological outcomes still need to be further investigated with a large-scale, multi-centre, prospective study.

Although multiple methods of bystander FB removal in FBAO victims have been described in the literature, there is little evidence showing that one way is better than the others in the real world. Most of the previous studies were simulation-based and examined the efficacy of FB removal manoeuvres performed by non-elderly healthy volunteers on recently deceased persons or manikins. ${ }^{28}{ }^{29}$ However, the majority of FBAO victims are elderly patients, witnesses to these events are likely elderly family members or friends and efficacy of FB removal manoeuvres performed by elderly individuals need to be investigated. In this study, we collect basic demographic information for bystanders to examine the efficacy of the FB removal manoeuvres.

In addition to the efficacy of the manoeuvres, we will investigate potential complications from these manoeuvres. Unfortunately, studies on complications from FB removal manoeuvres are scarce and mainly limited to case series and case reports. ${ }^{17-19}$ Due to the absence of a large cohort study of FBAO that documents removal manoeuvres, the exact incidence of complications is unknown. As described above, it is extremely rare for EMS personnel to terminate resuscitation efforts in the field and patients are typically transported to the ED even when resuscitations seem futile (eg, asystole in the field).$^{30}$ Also, autopsy imaging is often performed in the ED when patients die in the ED. ${ }^{31}$ These unique situations in Japan will provide us with the opportunity to describe a more comprehensive set of characteristics of complications from FB removal manoeuvres.

Our results will be meaningful for not only emergency physicians and emergency medical services but also for public health policymakers. If bystander FB removal has a critical role in the prognosis of FBAO victims and at the same time, there is a significant barrier for a bystander to perform FB removal successfully (eg, age of the bystander), an alternative method of bystander FB removal might need to be developed and taught in the future. For out-of-hospital cardiac arrest, the use of publicly accessible AEDs significantly improved survival and neurological outcomes. A similar approach, that is, to introduce a publicly accessible FB removal device might also need to be considered. 


\section{ETHICS AND DISSEMINATION}

The study will be conducted in accordance with the Declaration of Helsinki. This study received research ethics approval from Nippon Medical School Hospital (B-2019-019). Research ethics approval will be obtained from all participating sites before entering patients into the registry. The study was registered at the University Hospital Medical Information Network (UMIN) Clinical Trials Registry. The results will be disseminated through publications in peer-reviewed journals and presentations at scientific conferences.

\section{Author affiliations}

${ }^{1}$ Department of Emergency Medicine, University of New Mexico, Albuquerque, New Mexico, USA

${ }^{2}$ Department of Traumatology and Acute Critical Medicine, Osaka University Graduate School of Medicine, Suita, Osaka, Japan

${ }^{3}$ Department of Emergency and Critical Care Medicine, Nippon Medical School Hospital, Bunkyo-ku, Tokyo, Japan

${ }^{4}$ Department of Critical Care Medicine, Osaka Habikino Medical Center, Habikino, Osaka, Japan

${ }^{5}$ Department of Emergency Medicine, Osaka Police Hospital, Osaka, Japan ${ }^{6}$ Department of Emergency and Critical Care Medicine, Nippon Medical School Musashi Kosugi Hospital, Kawasaki, Kanagawa, Japan

${ }^{7}$ Department of Emergency Medicine, Aidu Chuo Hospital, Aizuwakamatsu, Fukushima, Japan

${ }^{8}$ Department of Emergency Medicine, Japanese Red Cross Ashikaga Hospital, Ashikaga, Tochigi, Japan

Contributors TN conceived the research idea, designed the study protocol and is the principal investigator. Yl and KS-H conceived and developed the study protocol. SNagata, TT, YY, TH, RM, SNakao, SY, HY and TS organised the study. TN, DA and CC drafted and revised the manuscript, and all authors critically reviewed the content and approved the final manuscript.

Funding This study is supported by the Japanese Association for Acute Medicine. Competing interests None declared.

Patient and public involvement Patients and/or the public were not involved in the design, or conduct, or reporting, or dissemination plans of this research.

Patient consent for publication Not required.

Provenance and peer review Not commissioned; externally peer reviewed.

Open access This is an open access article distributed in accordance with the Creative Commons Attribution Non Commercial (CC BY-NC 4.0) license, which permits others to distribute, remix, adapt, build upon this work non-commercially, and license their derivative works on different terms, provided the original work is properly cited, appropriate credit is given, any changes made indicated, and the use is non-commercial. See: http://creativecommons.org/licenses/by-nc/4.0/.

\section{ORCID iD}

Tatsuya Norii http://orcid.org/0000-0001-7428-5625

\section{REFERENCES}

1 National Safety Council. Report on injuries. injury facts, 2017. Available: https://injuryfacts.nsc.org/ [Accessed 28 Oct 2019]

2 Landoni G, Morselli F, Silvetti S, et al. Pizza in adults and grape in children are the most frequent causes of foreign body airway obstruction in Italy. A national media-based survey. Resuscitation 2020;149:141-2.

3 Pavitt MJ, Nevett J, Swanton LL, et al. London ambulance source data on choking incidence for the calendar year 2016: an observational study. BMJ Open Respir Res 2017;4:e000215.

4 Chung $\mathrm{C}-\mathrm{H}$, Lai C-H, Chien W-C, et al. A population-based study of inpatients admitted due to suffocation in Taiwan during 2005-2007. Accid Anal Prev 2013;50:523-9.
5 Igarashi Y, Norii T, Sung-Ho K, et al. Seasonal choking in Japan: Japanese rice cake (mochi), ehomaki, and beans for Setsubun. Resuscitation 2020;150:90-1.

6 Ministry of Health, Labor and Welfare in Japan. Report of vital statistics in 2018. Available: https://www.mhlw.go.jp/toukei/saikin/ hw/jinkou/geppo/nengai18/dl/gaikyou30.pdf [Accessed 28 Oct 2019].

7 Park GJ, Song KJ, Shin SD, et al. Timely bystander CPR improves outcomes despite longer EMS times. Am J Emerg Med 2017;35:1049-55.

8 Weaver WD, Cobb LA, Hallstrom AP, et al. Factors influencing survival after out-of-hospital cardiac arrest. J Am Coll Cardiol 1986;7:752-7.

9 Wolf DA. Heimlich trauma: a violent maneuver. Am J Forensic Med Pathol 2001;22:65-7.

10 Becker LB, Aufderheide TP, Geocadin RG, et al. Primary outcomes for resuscitation science studies: a consensus statement from the American heart association. Circulation 2011;124:2158-77.

11 Takeda Y, Ochi G, Hatanaka T, et al. An analysis of foreign body removal from airway by EMS personnel and laypersons, 2000. Available: https://plaza.umin.ac.jp/ GHDNet/00/kajiti2.htm [Accessed 12 Apr 2020].

12 Luczak A. Effect of body position on relieve of foreign body from the airway. AIMS Public Health 2019;6:154-9.

13 Luczak A. Head-down self-treatment of choking. Resuscitation 2016;103:e13.

14 Igarashi Y, Norii T, Sung-Ho K, et al. Life-Threatening foreign body airway obstruction: case series and new classification proposal. Am J Emerg Med 2019.

15 Hodge SE, Kilpatrick L, Zdanski C. An Innovative Approach to Airway Foreign Body Management in an Extremely Premature Neonate [published online ahead of print, 2019 Jun 26]. Laryngoscope 2019.

16 Nagata S, Kim S-H, Mizushima Y, et al. Airway obstruction due to sticky rice cake (mochi): a case series and review of the literature. Int J Emerg Med 2018;11:31-4.

17 Lee SL, Kim SS, Shekherdimian S, et al. Complications as a result of the Heimlich maneuver. J Trauma 2009;66:E34-5.

18 Fearing NM, Harrison PB. Complications of the Heimlich maneuver: case report and literature review. J Trauma 2002;53:978-9.

19 van der Ham AC, Lange JF. Traumatic rupture of the stomach after Heimlich maneuver. J Emerg Med 1990;8:713-5.

20 Pandharipande PP, Girard TD, Jackson JC, et al. LongTerm cognitive impairment after critical illness. N Engl J Med 2013;369:1306-16.

21 Riva G, Ringh M, Jonsson M, et al. Survival in Out-of-Hospital Cardiac Arrest After Standard Cardiopulmonary Resuscitation or Chest Compressions Only Before Arrival of Emergency Medical Services: Nationwide Study During Three Guideline Periods [published online ahead of print, 2019 Apr 1]. Circulation 2019.

22 Kiyohara K, Sakai T, Nishiyama C, et al. Epidemiology of out-ofhospital cardiac arrest due to suffocation focusing on suffocation due to Japanese rice cake: a population-based observational study from the Utstein Osaka project. J Epidemiol 2018;28:67-74.

23 Inamasu J, Miyatake S, Tomioka $\mathrm{H}$, et al. Cardiac arrest due to food asphyxiation in adults: resuscitation profiles and outcomes. Resuscitation 2010;81:1082-6.

24 Tipton MJ, Golden FSC. A proposed decision-making guide for the search, rescue and resuscitation of submersion (head under) victims based on expert opinion. Resuscitation 2011;82:819-24.

25 Szpilman D. Near-Drowning and drowning classification: a proposal to stratify mortality based on the analysis of 1,831 cases. Chest 1997;112:660-5.

26 Szpilman D, Bierens JJLM, Handley AJ, et al. Drowning. N Engl J Med 2012;366:2102-10.

27 Igarashi Y, Yokobori S, Yoshino Y, et al. Prehospital removal improves neurological outcomes in elderly patient with foreign body airway obstruction. Am J Emerg Med 2017;35:1396-9.

28 Langhelle A, Sunde K, Wik L, et al. Airway pressure with chest compressions versus Heimlich manoeuvre in recently dead adults with complete airway obstruction. Resuscitation 2000;44:105-8.

29 Ichikawa M, Oishi S, Mochizuki K, et al. Influence of body position during Heimlich maneuver to relieve supralaryngeal obstruction: a manikin study. Acute Med Surg 2017;4:418-25.

30 Norii T, Matsushima K, Miskimins RJ, et al. Should we resuscitate elderly patients with blunt traumatic cardiac arrest? analysis of national trauma registry data in Japan. Emerg Med J 2019;36:670-7.

31 Okuda T, Shiotani S, Sakamoto N, et al. Background and current status of postmortem imaging in Japan: short history of "Autopsy imaging (Ai)". Forensic Sci Int 2013;225:3-8. 